\title{
Vitamin B12 in pregnancy: Maternal and fetal/neonatal effects-A review
}

\author{
H. Van Sande, Y. Jacquemyn", N. Karepouan, M. Ajaji \\ Department of Obstetrics and Gynaecology, Antwerp University Hospital (UZA), Edegem, Belgium \\ Email: *Yves.Jacquemyn@uza.be
}

Received 27 March 2013; revised 28 April 2013; accepted 6 May 2013

Copyright (C) 2013 H. Van Sande et al. This is an open access article distributed under the Creative Commons Attribution License, which permits unrestricted use, distribution, and reproduction in any medium, provided the original work is properly cited.

\begin{abstract}
Vitamin B12 maintains normal folate metabolism which is essential for cell multiplication during pregnancy. No good data are available on what constitutes vitamin $B 12$ deficiency in pregnancy, nevertheless vitamin B12 deficiency is frequently reported in pregnancy due to inadequate dietary intake of vitamin B12 and a physiological decline of maternal vitamin B12 concentrations. This decline can be explained by the increased maternal metabolic rate and active transport by the placenta to the fetus. If the mother is deficient during pregnancy, the baby may have low serum vitamin B12 concentrations at birth [1]. Vitamin B12 deficiency during pregnancy is associated with preeclampsia, fetal growth restriction, preterm labor, neural tube defects, neonatal megaloblastic anemia and neonatal neurological symptoms. It has been suggested to improve vitamin B12 status of women in the periconceptional period by supplementation of vitamin B12, but no randomized studies on the outcome of such supplementation have been published.
\end{abstract}

Keywords: Vitamin B12; Pregnancy; Fetus

\section{INTRODUCTION}

Vitamin B12 deficiency is emerging as a growing public health problem and an increasing number of studies show that deficiency is commonly seen in pregnancy. This article reviews vitamin B12 deficiency during pregnancy, its effect on pregnancy, and the health of the offspring.

\section{METHODS}

A PubeMed search was performed in august 2011, and reviewed February 2013, with search terms: "vitamin B12 and pregnancy" "cobolamin and pregnancy", "vita-

\footnotetext{
"Corresponding author.
}

min B12 and fetus or neonate" "cobolamin and fetus or neonate" "vitamin B12 and lactation" "cobalamin and lactation".

\section{RESULTS}

Our search resulted in 2197 articles. Based on title and abstract 29 articles were selected relevant to our purpose [1]. Data from these studies are discussed.

\section{DISCUSSION}

\subsection{Basic Physiology}

Vitamin B12 or "cobalamin" is a water soluble vitamin that is only present in foods of animal origin such as meat, fish, dairy products and eggs. In supplements and fortified foods the synthetic form of vitamin B12, known as cyanocobalamin is used.

Cobalamin plays a crucial in DNA-synthesis and regulation and in the synthesis of fatty acids and energy production. It is metabolically related to folate as it is involved as a coenzyme in folate metabolism. Therefore vitamin B12 is also necessary for erythropoiesis and essential for normal neurodevelopment [2]. Cobalamin is involved as a coenzyme in 2 enzymatic reactions. In the first enzymatic reaction, methionine synthetize produces methionine from homocysteine in the cytosol. In the second enzymatic reaction adenosylcobalamin is involved as a coenzyme in the production of methylmalonylco-enzym-A (methylmalonyl-CoA) to succinyl-CoA in the mitochondrion. A vitamin B12 deficiency results in elevated concentrations of homocystein and methylmalonic acid in serum [3].

Vitamin B12 from dietary intake is bound to proteins and is released by hydrochloric acid that is secreted by the parietal cells from the stomach. The free form of vitamin B12 is then immediately bound to haptocorrins, which are glycoproteins secreted by the stomach and salivary glands. These haptocorrins protect vitamin B12 
from chemical denaturation in the stomach. The parietal cells secrete intrinsic factor that binds to vitamin B12 and enables its active absorption. When the contents of the stomach enter the duodenum, the haptocorrins become partly digested by the pancreatic proteases. This causes them to release their vitamin B12. Immediately after they are released, they get bound to intrinsic factor in the duodenum. The vitamin B12-intrinsic factor complex then proceeds to the ileum, where it is absorbed by specific ileal receptors [3].

\subsection{Vitamin B12 and Pregnancy}

Vitamin B12 maintains normal folate metabolism which is essential for cell multiplication, specifically in the rapidly dividing placental and fetal tissues. Together with folate and vitamin B6, it also functions as a coenzyme in DNA synthesis and numerous methylation reactions which occur in developing embryos [4].

Only limited data about transplacental transfer of B vitamins are available. It has been reported that the placenta synthesizes transcobalamin and contains transcobalamin receptors. In the intervilous space of the placenta, vitamin B12 and folate are sequestered so they can be distributed to the fetus. The fetus uses the available amount of vitamin B12 for biochemical reactions, but is not able to synthetize vitamin B12 [4].

From the first through the third trimester of pregnancy there is a gradual decline in the serum concentration of vitamin B12 due to hemodilution, hormonal changes, alterations in the concentration of vitamin B12 binding proteins, and placental transport of vitamin B12 to the fetus. The lowest concentration of vitamin B12 is seen at 32 weeks of pregnancy and before delivery it increases again to reach a normal level after birth. The concentration of the active part of vitamin B12, holotranscobalamin, remains unchanged during pregnancy [5].

There are no reference values available for vitamin B12 status in pregnant women. Hence, the reference values for nonpregnant individuals are often used to assess the vitamin B12 status during pregnancy [6]. It has been suggested that women should start pregnancy with serum vitamin $\mathrm{B} 12$ concentrations of $221 \mathrm{pmol} / \mathrm{L}$ and that a concentration above $295 \mathrm{pmol} / \mathrm{L}$ is desirable to minimalize the risk of developing complications [7]. These proposed thresholds are not based on prospective studies evaluating pregnancy outcome. As the physiologic adaptation seems to be a lower vitamin B12 level during pregnancy, using cut off values of the non-pregnant populations will artificially increase the number of pregnant women diagnosed with vitamin B12 deficiency, but we do not know whether this has any clinical relevance. Vitamin B12 levels have been shown to differ between racial/ethnic groups and the clinical consequences, if any, of this are still not understood [8].
Micronutrient deficiencies during pregnancy have been shown to have serious implications on the developing foetus. A vitamin B12 deficiency is seen worldwide lately but not all patients with a deficiency develop symptoms. Hematological signs of vitamin B12 deficiency only occur in very severe deficiency. Globally the data clearly show that megaloblastic anemia does not accompany the level of vitamin B12 deficiency that is so prevalent in developing countries.

Vitamin B12 status during pregnancy is critical since maternal vitamin B12 deficiency can affect the pregnancy outcome for both mother and the offspring. For women who want to get pregnant, a vitamin B12 deficiency means an increased risk of developing preeclampsia, intra-uterine growth retardation, preterm labor $[7,9]$. Children of deficient mothers are at risk for low birth weight $[4,7,10]$. Recent studies have also found an association between low vitamin B12 status in mothers and neural tube defect. This suggests an increased risk for birth defects when starting pregnancy with a deficient or inadequate vitamin B12 status, although this association clearly does not prove any causal relationship [7]. There might be also an association with increased adiposity which might lead to insulin resistance and gestational diabetes $[11,12]$.

All of these observations are based on a number of anecdotal studies. In these studies, cut off concentrations of vitamin B12 were different. They all use reference minimum concentrations used in non pregnant women, which variate from $180 \mathrm{pg} / \mathrm{ml}$ tot $350 \mathrm{pg} / \mathrm{m}$. Given the knowledge that there is a physiological dilution during pregnancy of vitamin B12, it is not clear in literature which minimal concentration of vitamin B12 really gives higher risks of complications during pregnancy.

\subsection{Breastfeeding and Vitamin B12}

A vitamin B12 deficiency during pregnancy can affect the health of the infant due to low vitamin B12 stores at birth and because of inadequate concentrations of vitamin B12 in breast milk [13]. Infants who are exclusively breastfed by mothers who have a low dietary intake of vitamin B12 such as vegans and vegetarians are therefore at risk for developing vitamin B12 deficiency possibly influencing cognitive and psychomotor development [14].

A severe vitamin B12 deficiency in the infant can cause neurological symptoms, including irritability, failure to thrive, apathy, anorexia and hematological symptoms such as megaloblastic anemia [15]. At a later age developmental regression, like impaired growth, gross motor function, poor school performance and other adaptive skills has been suggested to be a consequence of a poor maternal vitamin B12 status during pregnancy [1, $4,16]$. 


\subsection{Supplementation during Pregnancy and Breastfeeding}

Vitamin B12 status is usually assessed by measuring the total vitamin B12 concentration in serum and deficiency is currently defined as a concentration below $148 \mathrm{pmol} / \mathrm{L}$ (200 pg/mL), a marginal status is defined as $148-221$ $\mathrm{pmol} / \mathrm{L}$. Vitamin B12 deficiency can be caused by inadequate dietary intake or malabsorption of the vitamin from food. Vegetarians and vegans are considered to be at high risk for developing a vitamin B12 deficiency. Numerous studies on smaller population groups confirmed that both vitamin B12 intake and serum vitamin B12 concentrations increase progressively from vegans to lacto-ovo-vegetarians, to those who consume fish or some meat, to omnivores. Deficiency for vitamin B12 is not confined to these groups and occurs in any population [13].

According to a $2004 \mathrm{WHO}$ report $0.2 \mu \mathrm{g}$ /day of vitamin B12 needs to be added to the Estimated Average Requirement (EAR) for adults in pregnant women. This will result in an EAR of $2.2 \mathrm{mg} /$ day and a Recommended Nutrient Intake (RNI) of $2.6 \mathrm{mg}$ /day during pregnancy as absorption of nutrients is not $100 \%$. There is no consensus on the optimal intake during pregnancy. Some authors have suggested that an optimal dietary intake of vitamin B-12 during pregnancy should be at least $3 \mu \mathrm{g} / \mathrm{d}$ [17].

Breastfeeding women with adequate vitamin B12 status secrete approximately $0.4 \mu \mathrm{g} /$ day vitamin B12 in their milk, for this reason it has been suggested that lactating women need an extra $0.4 \mu \mathrm{g} /$ day vitamin B12 in addition to the normal adult requirement of $2.0 \mu \mathrm{g} /$ day. This results in a total EAR of $2.4 \mu \mathrm{g} /$ day and a RNI of $2.8 \mu \mathrm{g} /$ day during lactation. To prevent further depletion of vitamin B12 in lactating women due to breast feeding, it has been advised to provide multiple micronutrient supplements $[2,18]$. We were not able to find any prospective and/or randomized study comparing outcome of pregnancy and/or lactation in women using or not using vitamin B12 supplements.

\section{DISCUSSION}

Because of the consequences of a poor maternal vitamin B12 status on the health and development of the offspring, it seems necessary that mothers start their pregnancies with an adequate vitamin status. No studies shave definitively determined the best cut off value for maternal serum vitamin B12 level before conception during pregnancy and in the lactational period, nor has attention been paid to racial/ethnic differences in these serum levels. Theoretically supplementation with vitamin B12 could reduce the risk for congenital anomalies such as neural tube defects and improve infant health, but no prospective studies have been published. Although different authors have noted an association between low vitamin B12 in maternal serum and gestational and fetal/ neonatal complications, no trials proving a causal relationship or any trial demonstrating the efficacy of interventions with vitamin B12 supplements could been located. Such a trail should also evaluate potential harmful side effects.

\section{CONCLUSION}

Improvements in maternal nutritional status have the potential to improve health outcomes for infants. Because of the consequences of poor maternal vitamin status on the outcome of pregnancy and offspring, as a clinician it is important to pay attention to this topic when women come with a childbearing desire. Early detection of a vitamin B12 deficiency before becoming pregnant and prevention of maternal micronutrient deficiencies in the periconceptional period is a possible path to avoid some complications during pregnancy and reduce health problems in the infant. Before starting supplementation without any proven benefit or with potential harmful effects, we need randomized prospective studies to determine the effect of vitamin B12 supplementation in pregnancy and determine the optimal way and dose of administration if necessary.

\section{REFERENCES}

[1] Hay, G., Clausen, T., Whitelaw, A., Trygg, K., Carole, J., Tore, H. and Helga, R. (2010) Maternal folate and cobalamin status predicts vitamin status in newborns and 6-month-old infants. Journal of Nutrition, 140, 557-564. doi:10.3945/jn.109.117424

[2] Obeid, R. and Herrmann, W. (2005) Homocysteine. Folic acid and vitamin B12 in relation to pre- and postnatal health aspects. Clinical Chemistry and Laboratory Medicine, 43, 1052-1057. doi:10.1515/CCLM.2005.184

[3] Refsum, H. (2007) Folate, vitamin B12 and homocysteine in relation to birth defects and pregnancy outcome. British Journal of Nutrition, 854, 109-113.

[4] Molloy, A.M., Kirke, P.N., Brody, L.C., et al. (2008) Effects of folate and vitamin B12 deficiencies during pregnancy on fetal, infant, and child development. Food and Nutrition Bulletin, 29, 101-111.

[5] Lee, Y.K., Kim, H.S. and Kang, H.J. (2009) Holotranscobalamin as an indicator of vitamin B12 deficiency in gastrectomized patients. Annals of Clinical \& Laboratory Science, 39, 361-366.

[6] Dror, D.K. and Allen, L.H. (2012) Interventions with vitamins B6, B12 and $\mathrm{C}$ in pregnancy. Pediatric and Perinatal Epidemiology, 26, 55-74. doi:10.1111/j.1365-3016.2012.01277.x

[7] Krishnaveni, G.V., Hill, J.C. and Veena, S.R., et al. (2009) Low plasma vitamin B12 in pregnancy is associated with 
gestational "diabesity" and later diabetes. Diabetologia, 52, 2350-2358. doi:10.1007/s00125-009-1499-0

[8] Mursu, J., Robies, K., Harnack, L.J., Pack, K. and Jacobs Jr., D.R. (2011) Dietary supplements and mortality rate in older women, the Iowa women's health study. Archives of Internal Medicine, 171, 1625-1633. doi:10.1001/archinternmed.2011.445

[9] Hübner, U., Alwan, A., Jouma, M., Tabbaa, M., Schorr, H. and Hermann, W. (2008) Low serum vitamin B12 is associated with recurrent pregnancy loss in Syrian women. Clinical Chemistry and Laboratory Medicine, 46, 1265-1269. doi:10.1515/CCLM.2008.247

[10] Butler, C.C., Vidal-Alaball, J., Cannings-John, R., et al. (2006) Oral vitamin B12 versus intramuscular vitamin B12 for vitamin B12 deficiency: A systematic review of randomized controlled trials. Family Practice, 23, 279285. doi:10.1093/fampra/cml008

[11] Hure, A., Collins, C. and Smith, R. (2012) A longitudinal study of maternal folate and vitamin B12 status in pregnancy and postpartum, with the same infant markers at 6 months of age. Maternal and Child Health Journal, 16, 792-801.

[12] Saxena, S. and Carmel, R. (1987) racial differences in vitamin b12 levels in the United States. American Journal of Clinical Pathology, 88, 95-97.

[13] Allen, L.H. (2009) How common is vitamin B-12 deficiency? The American Journal of Clinical Nutrition, 89, 693S-696S. doi:10.3945/ajcn.2008.26947A

[14] Black, M.M. (2003) Micronutrient deficiencies and cognitive functioning. Journal of Nutrition, 133, 3927S$3931 \mathrm{~S}$.

[15] Obeid, R., Munz, W., Jäger, M., et al. (2005) Biochemical indexes of the $\mathrm{B}$ vitamins in cord serum are predicted by maternal $\mathrm{B}$ vitamin status. The American Journal of Clinical Nutrition, 82, 133-139.

[16] Dror, D.K. and Allen, L.H. (2008) Effect of vitamin B12 deficiency on neurodevelopment in infants: current knowledge and possible mechanisms. Nutrition Reviews, 66, 250-255. doi:10.1111/j.1753-4887.2008.00031.x

[17] Ray, J.G. and Blom, H.J. (2003) Vitamin B12 insufficiency and the risk of fetal neural tube defects. Quarterly Journal of Medicine, 96, 289-295. doi:10.1093/qjmed/hcg043

[18] Allen, L.H. (2005) Multiple micronutrients in pregnancy and lactation: An overview. The American Journal of Clinical Nutrition, 81, 1206S-1212S. 